\title{
Phalangeal Tuberculosis After Mallet Finger Treatment: A Case Report
}

\author{
Seyed Amir Mahlisha Kazemi Shishavan, ${ }^{1}$ and Ali Tabrizi ${ }^{2,}{ }^{*}$ \\ ${ }^{1}$ Department of Orthopedics, Shariati Hospital, Tehran University of Medical Sciences, Tehran, Iran \\ ${ }^{2}$ Department of Orthopedics, Imam Khomeini Hospital, Urmia University of Medical Sciences, Urmia, Iran \\ "Corresponding author: Ali Tabrizi, Department of Orthopedics, Imam Khomeini Educational Hospital, Urmia University of Medical Sciences, Moderres Ave., Urmia, Iran. Tel: \\ +98-9143130829, E-mail: ali.tab.ms@gmail.com
}

Received 2018 January 29; Accepted 2018 April 09.

\begin{abstract}
Introduction: Skeletal involvements are less reported in tuberculosis and even less likely observed in fingers. Fingers are rarely involved in adults and it often has been reported in children under 5 years old. Most likely, a recent condition in adult patients is required to provoke reactivation of bacilli lodged in the bone during the original mycobacteremia of primary infection.

Case Presentation: In this report, a 31 - year - old female patient, suffered from detached extensor tendon due to the fourth finger trauma, was diagnosed as a Mallet finger and treated by closed percutaneous pining is introduced. The patient had chronic swelling and progressive pain in the same finger for six months after treatment. The common anti - inflammatory and antibiotic therapy was not successful. Radiographic images of the ring finger demonstrated erosion and irregularity of the articular surfaces around the distal interphalangeal join (DIP). She expressed a history of untreated cough and exposure to people with tuberculosis. A positive tuberculosis (TB) skin test was determined with more than $10 \mathrm{~mm}$ induration. Treatment with anti-tuberculosis medication regimen was successful and continued for 12 months.

Conclusions: Skeletal tuberculosis should always be considered by physicians in endemic areas. A slow progression of the disease in skeletal involvement and lack of clinical suspicion can lead to misdiagnosis. Using anti - tuberclusis medications for an appropriate period is effective in the disease control and treatment of patients.
\end{abstract}

Keywords: Dactylitis, Lytic Lesion, Tuberculosis

\section{Introduction}

Tuberculosis is considered as a rare disease in Western countries. In contrast, it constitutes the most common and endemic diseases in Third World and developing countries (1). Compared to pulmonary tuberculosis, skeletal involvement is unusual, consisting of $10 \%$ to $15 \%$ of patients with tuberculosis $(1,2)$. Skeletal tuberculosis in the hands comprises a wide range of bone or soft tissue involvement, which leads to various clinical symptoms (3). There are few reports regarding skeletal tuberculosis in the hands and fingers. Finger and metacarpus involvement in tuberculosis is one of the rare extra pulmonary manifestations (3). Overall, $1 \%$ to $3 \%$ of the skeletal tuberculosis occur in hands and fingers (3). Diagnosis of osseous tuberculosis, in most cases, is delayed due to the paucibacillary lesions and the negative smears (4). This report introduced a patient who was presented with a chronic and painful swelling in the fourth finger following mallet finger fixation that was attributed to phalangeal tuberculosis.

\section{Case Presentation}

A31-year-old rural woman with a history of chronic inflammation and painful range of motion in her (left) ring finger, for the past six months, was referred to the orthopedic clinic. These symptoms were presented following the surgical fixation of the Mallet finger, secondary to extensor tendon avulsion injury. Percutaneous pinning had been applied in a hyper extension position in the other center. The complication of dactylitis associated with progressive swelling in the finger was reported one month after removing the pin (Figure 1). No history of previous illness was noted. She only provided a history of intermittent cough several years ago as well as exposure to relatives with tuberculosis infections. Movement of the distal interphalangeal joint (DIP) was painful and severely restricted range of motion occurred progressively. At first she was treated imperatively with oral antibiotics including cephalexin (500 $\mathrm{mg} / \mathrm{q} 4 \mathrm{~h}$ ) and ciprofloxacin (500 mg/q2h) due to likely postoperative infection. However, no response to treatment was observed. Laboratory tests revealed that white blood cell (WBC) count was $8600 / \mathrm{mm}^{3}$ and erythrocyte sedimen- 


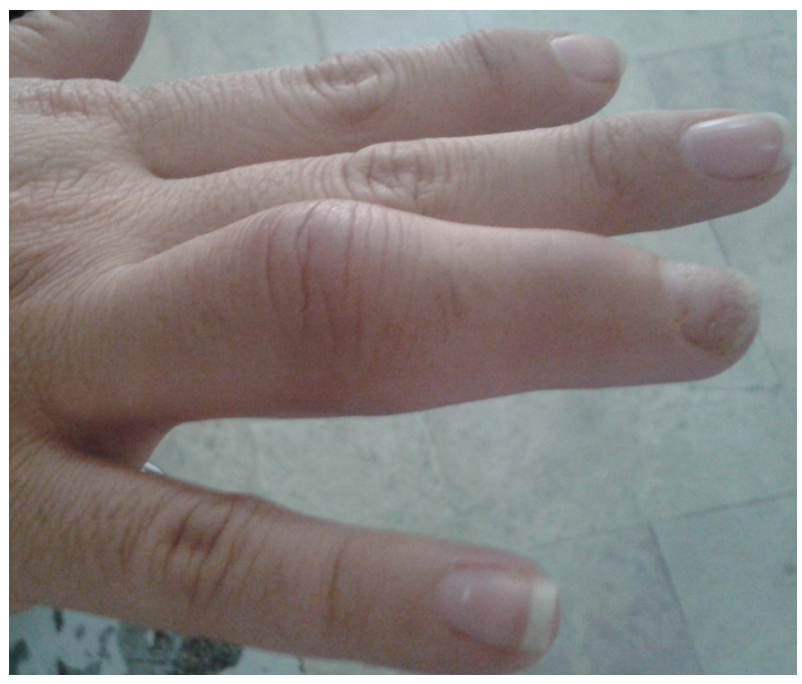

Figure 1. Clinical Photograph of a Dactylitis Swollen in Fourth Finger

tation rate (ESR) was 60. In the finger radiography, erosion was observed in DIP joint and kissing lesion was seen on the joint surface (Figure 2). The patient was a candidate for a biopsy of the lesion. DIP joint was opened through the volar approach. Operative exploration of the fourth finger demonstrated friable bone associated with grayish white necrosis material with soft tissue consistency. Culture of the sample was negative for bacteria or other microorganisms. Histology studies showed inflammatory granulomatous tissues with caseous necrosis. Tuberculosis (TB) skin test (PPD) was done and induration was measured more than $10 \mathrm{~mm}$ so that positive skin test was determined. According to histopathological findings, patient history, and positive skin test, the diagnosis of phalangeal tuberculosis dactylitis was recognized. She began taking chemotherapy for the tuberculosis - induced infection. Anti - tuberculosis treatment regimen including isoniazid, rifampicin, pyrazinamide, and streptomycin was prescribed for two months and was continued with rifampicin and isoniazid for 10 months. After a 12 - month follow - up, a maximal response to treatment was observed, including a significant decrease in the size of finger and improvement of lytic lesions with sclerosis margins. The pain was relieved and limitation of movement was disappeared.

\section{Discussion}

Mycobacterium tuberculosis can affect any part of the body. Mycobacterium tuberculosis - induced infection is extra pulmonary in $10 \%$ to $15 \%$ of the cases and it occurs in the skeletal system in $10 \%$ of the cases (5). Tuberculo-

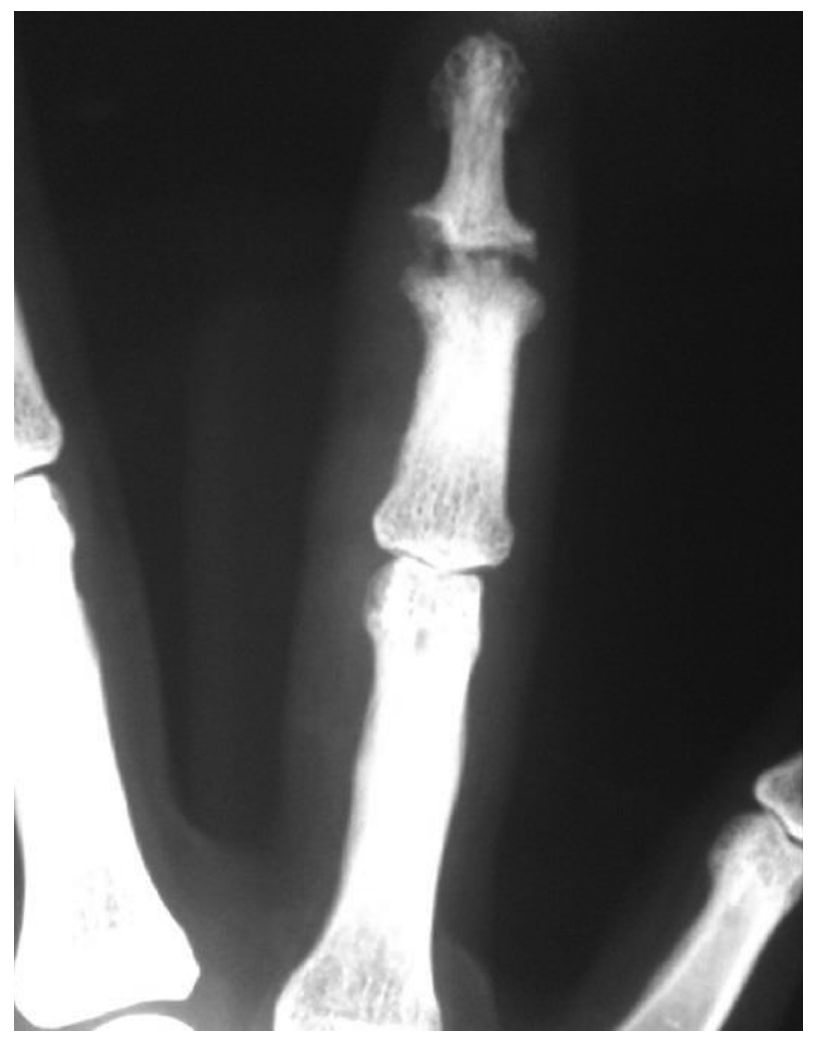

Figure 2. Radiography of the Fourth Finger with Erosions in DIP Joint, Lytic Lesions and Kissing Lesion

sis - induced tenosynovitis is reported as skeletal involvements in less than $1 \%$ of the cases and it has been reported extremely rare, as well. Early diagnosis and proper treatment is very important to keep the performance. Skeletal infection caused by the bacteria progresses slowly, leading to difficulties in early diagnosis (3). Late diagnosis can lead to the poor functional outcome (3). Pain and swelling are the most common manifestations of finger tuberculosis involvement (3). Tuberculosis in the fingers is seen typically in the phalangeal bones and interphalangeal joints. Finger involvement has been reported extremely rare in individuals over five years (6).

In the studied patient, chronic pain and swelling of the finger, with no response to the common antibiotics and nonsteroidal anti - inflammatory drugs, can be considered as a helpful sign to aware the surgeon. It has been stated that trauma plays an important role in the reactivation of tuberculosis (7). In the presented patient, trauma of the finger and a history of percutaneous pinning used for fixation of mallet finger can be counted as a reason for mycobacterium tuberculosis infection. The exact mechanism of how tuberculosis enters the skeletal system is still un- 
known. Although, it is estimated that it affects skeletal system by hematogenous dissemination following to pulmonary involvement or by direct inoculation $(6,7)$. Proximal phalanx of the second and third fingers have been more reported as dactylitis $(6,7)$. Erosion on the joint surface and dactylitis associated with chronic pain and swelling are the main presentations in the fingers tuberculosis infection. Histopathological studies can help in diagnosis. Differential diagnosis can have similar involvements including pyogenic osteomyelitis, osteoid osteoma, chondroma, synovitis sarcoidosis, and other granulomatous infections (7). It is probable that in patients with skeletal tuberculosis, concurrent pulmonary symptoms are not observed and merely there is a history of respiratory symptoms in the past. According to a report by Elmi et al., no pulmonary symptoms were observed in tuberculosis infection in a patient with a lytic lesion in the medial condyle of the femur (5). In the patient under study, there was also no pulmonary symptoms or respiratory system involvement. The chest radiograph was normal, which makes the diagnosis of tuberculosis really difficult. The regional tuberculosis infection of the hand can only be diagnosed through clinical suspicion and based on the symptoms.

Tuberculosis - induced dactylitis should be treated over a long-term, using anti - tuberculosis drugs. Treatment by isoniazid, rifampicin, pyrazinamide, and streptomycin for two months should be continued by using rifampicin and isoniazid for 10 to 14 months (7). According to the studies, skeletal involvement of tuberculosis needs to be treated longer. The mentioned treatment was very successful in our patient. Based on a study by Kotwal et al., in $75 \%$ of cases with tuberculosis hand involvement, medical treatment can provide a favorable clinical outcome (3).

\subsection{Conclusion}

Skeletal tuberculosis in endemic areas should always be considered by physicians. The slow progression and lack of clinical suspicion can lead to misdiagnosis. Using anti-tuberculosis drugs with an appropriate length is effective in the disease control and treatment of patients.

\section{Footnote}

Conflict of Interest: There is no conflict of interest to be reported.

\section{References}

1. Raviglione MC, Snider DJ, Kochi A. Global epidemiology of tuberculosis. Morbidity and mortality of a worldwide epidemic. JAMA. 1995;273(3):220-6. [PubMed: 7807661].

2. Hakimi M, Hashemi F, Mirzaie AZ, Pour AH, Kosari H. Tuberculous osteomyelitis of the long bones and joints. Indian $\mathrm{J} \mathrm{Pe}$ diatr. 2008;75(5):505-8. doi: 10.1007/s12098-008-0078-6. [PubMed: 18537013].

3. Kotwal PP, Khan SA. Tuberculosis of the hand: clinical presentation and functional outcome in 32 patients. $J$ Bone Joint Surg Br. 2009;91(8):1054-7. doi: 10.1302/0301-620X.91B8.22074. [PubMed: 19651833].

4. Agarwal S, Caplivski D, Bottone EJ. Disseminated tuberculosis presenting with finger swelling in a patient with tuberculous osteomyelitis: a case report. Ann Clin Microbiol Antimicrob. 2005;4:18. doi: 10.1186/1476-0711-4-18. [PubMed: 16269085].

5. Elmi A, Tabrizi A, Tolouei FM. Skeletal tuberculosis presenting as a small cystic lesion in the medial femoral condyle. Arch Bone Jt Surg. 2013;1(2):112-5. [PubMed: 25207301].

6. Abebe W, Abebe B, Molla K, Alemayehu T. Tuberculous Dactylitis: An Uncommon Presentation of Skeletal Tuberculosis. Ethiop J Health Sci. 2016;26(3):301-3. [PubMed: 27358553].

7. Sbai MA, Benzarti S, Sahli H, Sbei F, Maalla R. Osteoarticular tuberculosis dactylitis: Four cases. Int J Mycobacteriol. 2015;4(3):250-4. doi: 10.1016/j.ijmyco.2015.05.006. [PubMed: 27649874]. 